\title{
33 Target Tracking Using a 2D Radar
}

\author{
Marelize Kriel and Herman le Roux
}

\section{CONTENTS}

33.1 Introduction

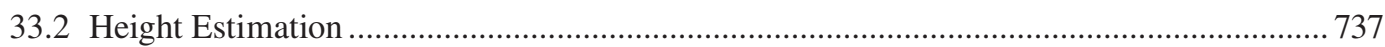

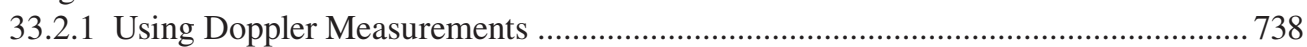

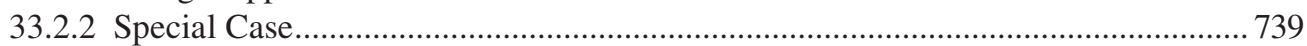

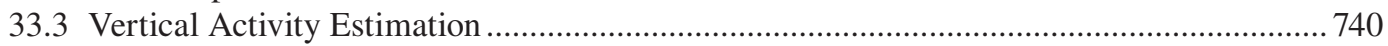

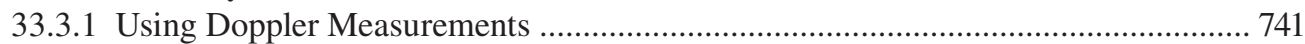

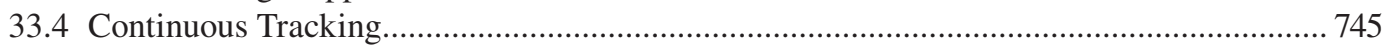

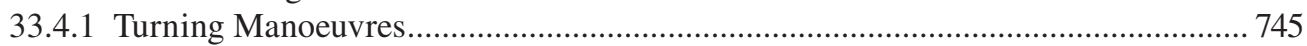

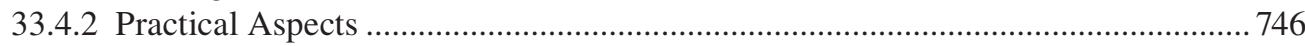

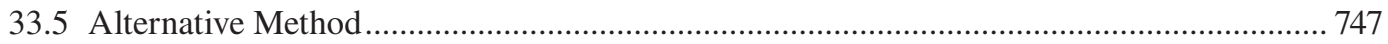

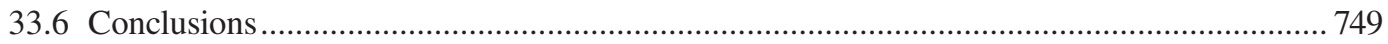

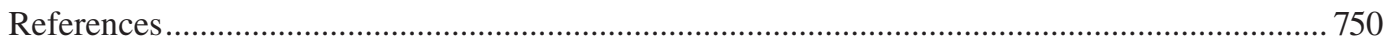

\subsection{INTRODUCTION}

This chapter briefly outlines a few mathematical techniques to track targets in $3 \mathrm{D}$ using a $2 \mathrm{D}$ radar. 2D radars are relatively cheap and efficient sensors that often form the first line of defence in airspace control. In military applications they are often used as early-warning devices because they can detect approaching enemy aircraft or missiles at great distances. In case of an attack, early detection of the enemy is vital for a successful defence against attack. Depending on the threat evaluation of tracked aircraft the tracking process is passed along to 3D search radars or fire control tracking radars once it comes into range of those sensors. A key component in the above hierarchy is the threat evaluation component. It relies on many factors such as angle of incidence towards defended assets, time to approach to defended asset, speed of target and so forth. The normal 2D radar provides range and azimuth but the altitude of the target is omitted. This can be an important consideration as aircraft altitude limits the attack profiles a target can fly [1].

\subsection{HEIGHT ESTIMATION}

The current literature regarding height estimation restricts itself to computations involving two or more $2 \mathrm{D}$ radars where the height can be completely determined by simple geometric computations. In this section we present some mathematical methods to infer aircraft altitude from two updates given by a single 2D radar [2]. A single 2D radar source cannot directly determine the altitude of aircraft, thus naturally, the method presented here is either coupled with a number of assumptions and limitations or is a mere approximation. The terms height and altitude are used interchangeably. Height often refers to the height of an aircraft above ground level, and altitude the height of the aircraft above mean sea level. The proposed techniques do not consider terrain, 


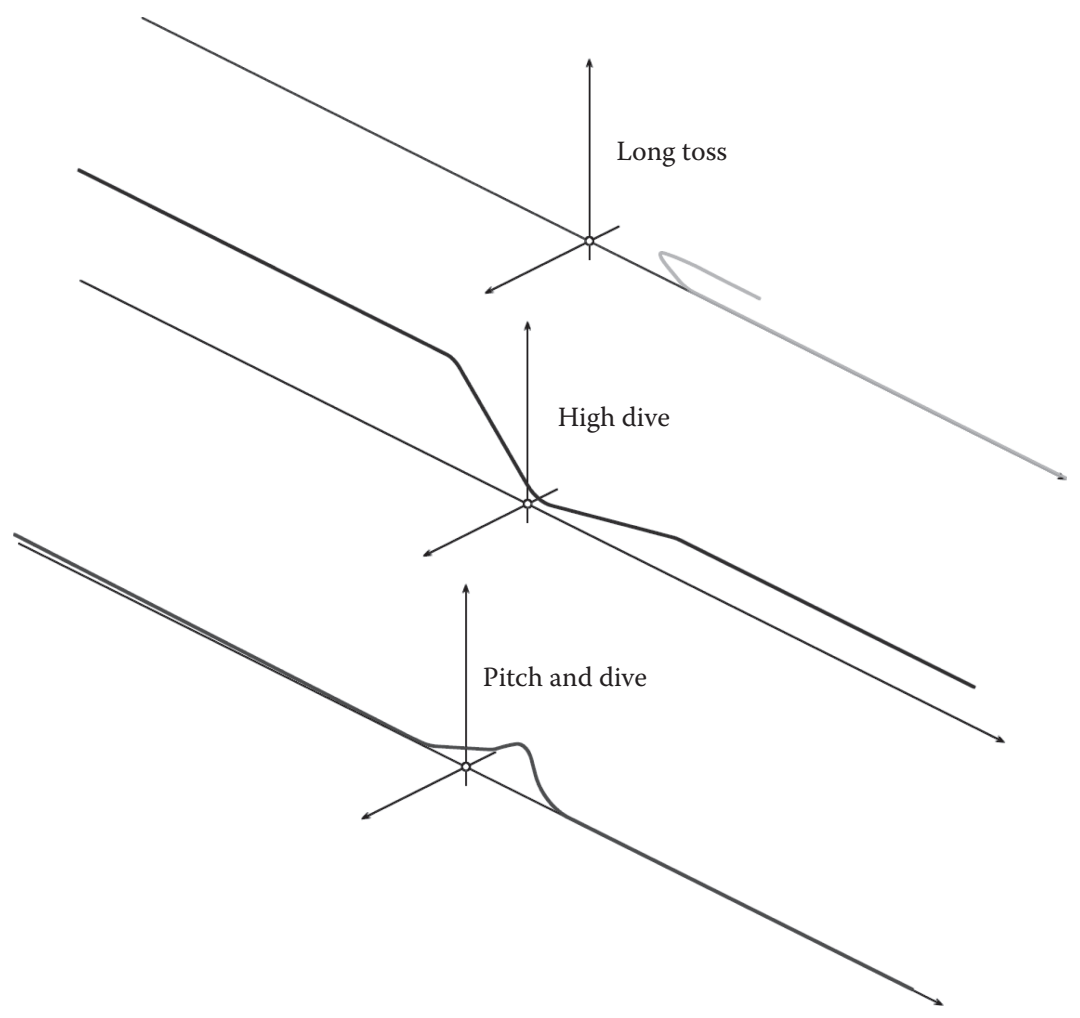

FIGURE 33.1 Flight profile examples.

terrain height, or height above mean sea level, but rather the difference in height of the sensor and observed aircraft.

It should also be noted that if the aircraft is flying perfectly tangential to the radar beam, then the radial speed component is zero and it is impossible to estimate its altitude. Conversely, however, it is more accurate to determine the altitude an aircraft flying at great speeds at a $45^{\circ}$ angle to the radar beam, than a slow flying aircraft that is flying towards the radar. The aircraft speed is instrumental in determining the aircraft altitude. The accuracy to which these speeds are known is directly proportional to the accuracy to which the altitude can be determined. Knowledge of aircraft speed can be obtained in a variety of ways. For example, due to the volatile nature of their payload, the speed at which bombers fly is usually controlled by doctrine, similarly cruise missiles fly at known speeds. We will make use of the three known flight profiles depicted in Figure 33.1 as examples. The defended asset as well as the sensor is located at the origin.

In the discussion that follows we will make use of two sequential sensor readings at time $t_{1}$ and $t_{2}$. The given data sets will consist of a slant range and azimuth reading, denoted $\left(r_{1}, \theta_{1}\right)$ and $\left(r_{2}, \theta_{2}\right)$. If the aircraft speed, denoted $v_{2}$, is known then we can easily determine the distance travelled between time $t_{1}$ and time $t_{2}$ as $u_{2}=v_{2}\left(t_{2}-t_{1}\right)$. In the figures (Figure 33.2) that follow we will, without loss of generality, assume that $r_{1} \geq r_{2}$.

\subsubsection{Using Doppler Measurements}

Modern 2D radar sensors allow Doppler measurements of one (radial) component of the velocity of the aircraft it is observing. In other words, Doppler measurements do not give us the velocity vector, $\vec{v}_{2}$, but only the magnitude of its radial component, denoted $\hat{v}_{2}$, at time $t_{2}$. From the measured radial 


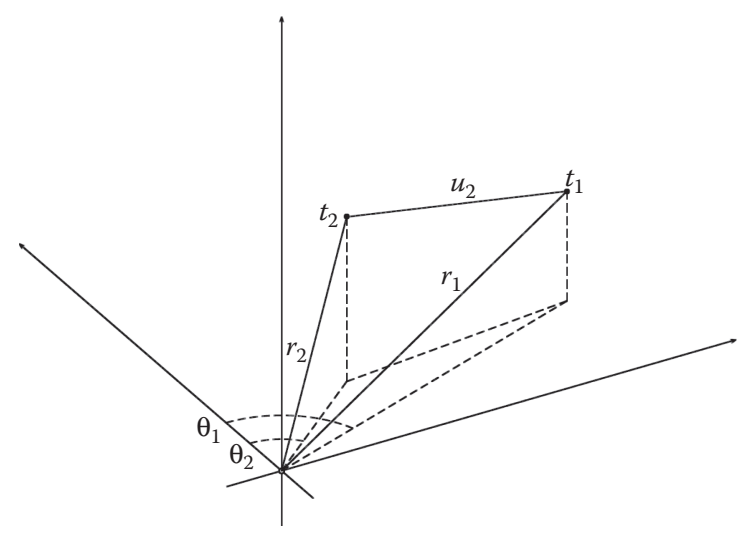

FIGURE 33.2 Radar tracking in 2D.

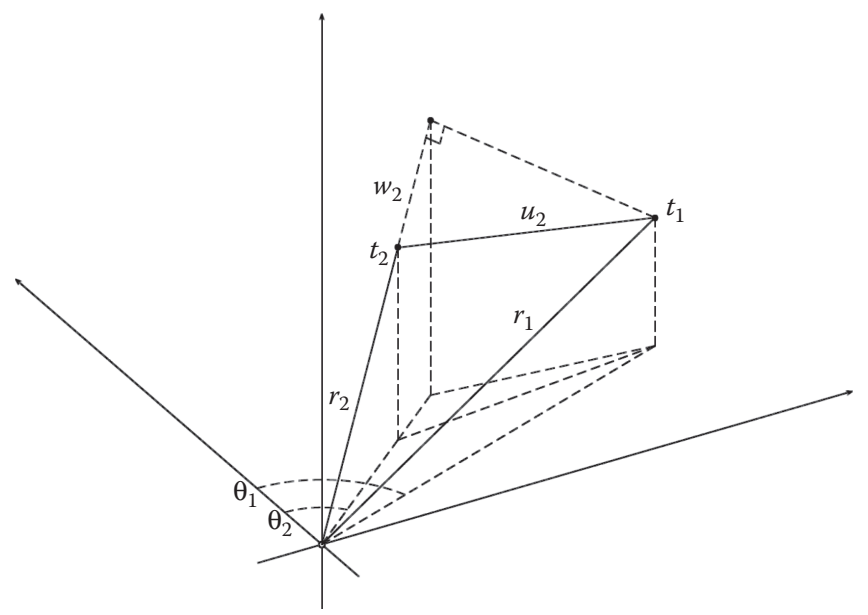

FIGURE 33.3 Radar tracking in 2D with Doppler data.

speed $\hat{v}_{2}$ we can easily determine the radial distance travelled $w_{2}=\hat{v}_{2}\left(t_{2}-t_{1}\right)$ and from that the total distance travelled is easily obtainable:

$$
u_{2}=\sqrt{r_{1}^{2}-\left(r_{2}+w_{2}\right)^{2}+w_{2}^{2}}
$$

It is clear from Figure 33.3 that the height can still not be directly calculated, even with Doppler measurements at hand, since the relations between these known values are valid at any height. In short, without any height-dependent data, there is no general way to directly compute the height.

\subsubsection{Special Case}

If we make an assumption that the aircraft is flying radially towards/away from the radar at level height and known speed (Figure 33.4), then we can compute the height with simple trigonometry as

$$
h_{1}=h_{2}=r_{1} \sqrt{1-\left(\frac{r_{2}^{2}-r_{1}^{2}-u_{2}^{2}}{-2 r_{1} u_{2}}\right)^{2}}
$$




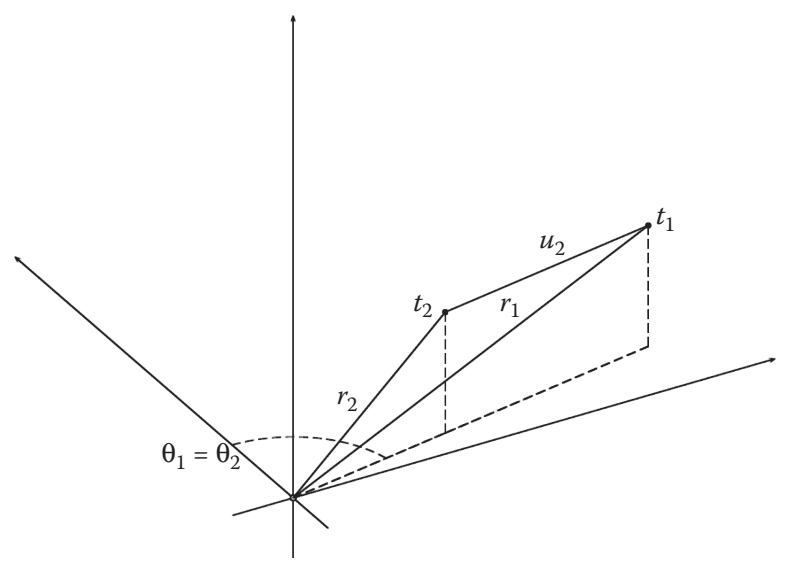

FIGURE 33.4 Simple case.

We will refer to this method as the AASC (Altitude Approximation via Simple Case) method to find an approximation to $h_{1}$ without knowing whether or not the assumptions of radial, straight flight hold true. The accuracy of this approximation is entirely dependent on the flight profile of the aircraft as illustrated in Figure 33.5. The model is usable without Doppler data as long as we have an accurate estimate of the speed of the aircraft, or more precisely $u_{2}$ so it seems natural to proceed with a sensitivity analysis to see how sensitive this model is to changes in the given $u_{2}$.

Assume that the aircraft is flying level and radially towards the sensor. We have $0<h=h_{1}=h_{2}<r_{1}$ and $\theta=\theta_{1}-\theta_{2}=0$. Thus

$$
u=u_{2}=\sqrt{r_{1}^{2}+r_{2}^{2}-2 h^{2}-2 \sqrt{r_{1}^{2}-h^{2}} \sqrt{r_{2}^{2}-h^{2}}}
$$

Differentiation yields

$$
\frac{\partial h}{\partial u}=\sqrt{r_{1}^{2}-h^{2}} \sqrt{r_{2}^{2}-h^{2}}\left(h \sqrt{r_{1}^{2}+r_{2}^{2}-2 h^{2}-2 \sqrt{r_{1}^{2}-h^{2}} \sqrt{r_{2}^{2}-h^{2}}}\right)^{-1}
$$

from which it is clear that

$$
\frac{\partial h}{\partial u} \rightarrow \infty \text { as } h \rightarrow 0 \quad \text { and } \quad \frac{\partial h}{\partial u} \rightarrow 0 \text { as } h \rightarrow r_{1}
$$

This implies that as the aircraft's height approaches zero, the estimated value of $h$ will become infinitely sensitive to changes in $u$ and as the aircraft's height approaches its range, the estimated height $h$ will become infinitely insensitive to changes in $u$.

\subsection{VERTICAL ACTIVITY ESTIMATION}

Understanding the vertical activity of an aircraft enables airspace control to predict aircraft intent which means more accurate situation awareness. The problem of estimating the behaviour of an aircraft is well known with applications in airspace control and threat evaluation.

The existing literature typically makes use of two or more separate radars to compute aircraft altitude. The advantages are immediately obvious as an aircraft can be tracked in 3D and the flight path can be compared to known flight profiles to offer a strong basis for threat evaluation. 


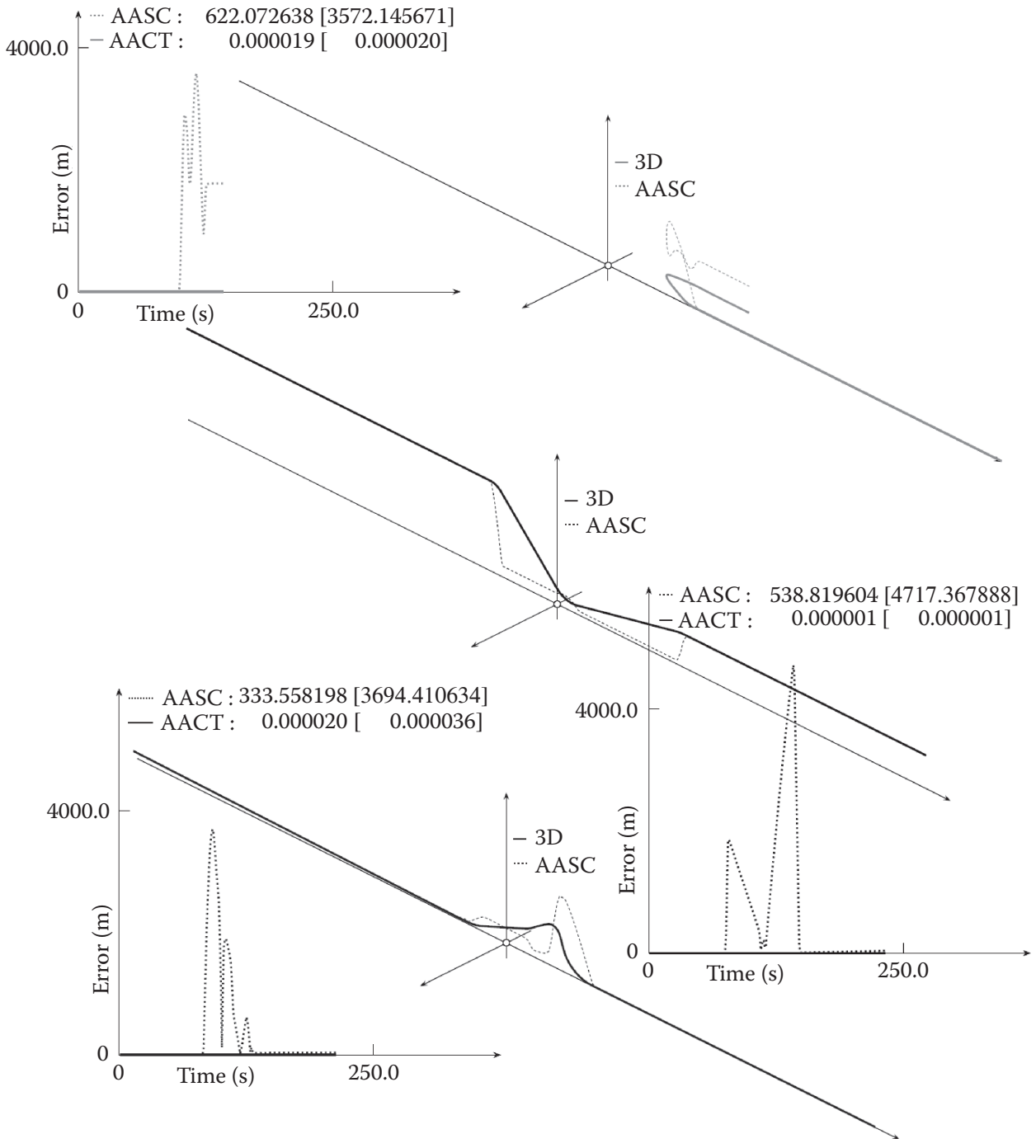

FIGURE 33.5 Height approximation using the AACT method.

\subsubsection{Using Doppler Measurements}

Vertical manoeuvres are easily and accurately recognizable when we have Doppler data available. Furthermore, it will give us a method to track the target in 3D.

If we have a relatively accurate estimation for $h_{1}$, then we can compute $h_{2}$ by solving the equation

$$
-w_{2}=r_{2}-r_{1} \cos \left(\theta_{1}-\theta_{2}\right) \cos \left(\epsilon_{1}\right) \cos \left(\epsilon_{2}\right)-r_{1} \sin \left(\epsilon_{1}\right) \sin \left(\epsilon_{2}\right)
$$

for $\epsilon_{2}$ once we know the value of $\epsilon_{1}$ as illustrated in Figure 33.6. For simplicity, we define

$$
\begin{array}{cl}
c_{1} \sin \left(\epsilon_{2}\right)+c_{2} \cos \left(\epsilon_{2}\right)+c_{3}=0 \quad \text { where } & c_{1}=r_{1} \sin \left(\epsilon_{1}\right)=h_{1} \\
& c_{2}=r_{2} \cos \left(\theta_{1}-\theta_{2}\right) \cos \left(\epsilon_{1}\right) \\
& c_{3}=-w_{2}-r_{2}
\end{array}
$$




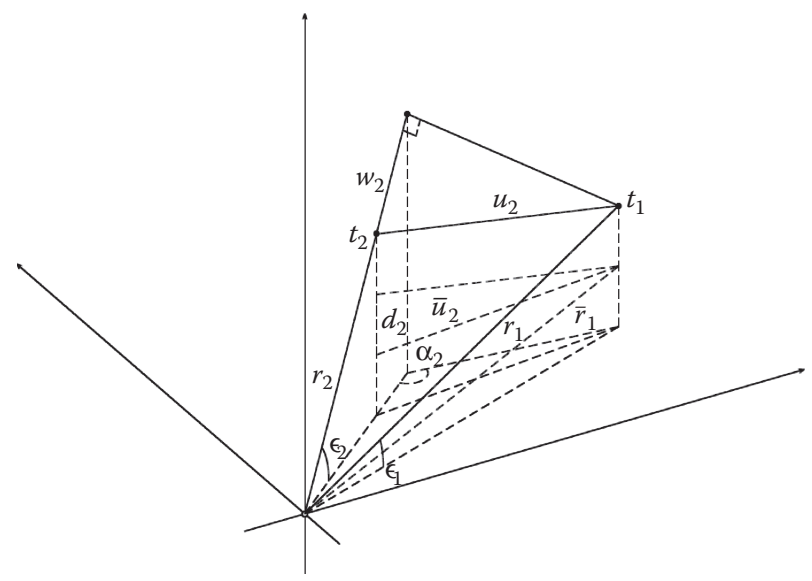

FIGURE 33.6 Calculation of change in altitude.

Then for $c_{1}^{2}+c_{2}^{2}-c_{3}^{2} \geq 0$ we have

$$
\epsilon_{2}=\left\{\begin{array}{l}
\epsilon_{2: 1}=2 \operatorname{atan}\left(\frac{c_{1}-\sqrt{c_{1}^{2}+c_{2}^{2}-c_{3}^{2}}}{c_{2}-c_{3}}\right) \text { if } \alpha_{2}>90^{\circ} \text { and } \\
\epsilon_{2: 2}=2 \operatorname{atan}\left(\frac{c_{1}-\sqrt{c_{1}^{2}+c_{2}^{2}-c_{3}^{2}}}{c_{2}-c_{3}}\right) \text { otherwise. }
\end{array}\right.
$$

We do not have enough information to calculate the projected angle $\alpha_{2}$ so we define two functions covering all possible projection angles.

$$
h_{2: 1}(h)=r_{2} \sin \left(\epsilon_{2: 1}\right) \quad \text { and } \quad h_{2: 2}(h)=r_{2} \sin \left(\epsilon_{2: 2}\right)
$$

where $\epsilon_{2: 1}$ and $\epsilon_{2: 2}$ is computed as above with $h_{1}=h$. We start by correcting the given initial height $\mathrm{h}$ if the error in range $\gamma(h)=\left|r_{1}-\bar{r}_{1}(h)\right|$ is too big (e.g., $>0.001$ ). We do this by adjusting the fixed height $h$ to

$$
h=\left\{\begin{array}{l}
h+\gamma(h) \\
h-\gamma(h)
\end{array}: \gamma(h \pm \gamma(h))\right. \text { is a minimum. }
$$

and continue to do so until $\gamma(h) \leq 0.001$.

Accuracy can further be increased by making this threshold smaller but at a computational cost. The absolute difference in height $d_{2}=\left|h_{2}-h_{1}\right|$ can be computed by projecting coordinates onto a plane of fixed height $h$ and using the Euclidean distance $\bar{u}_{2}(h)$ between the projected coordinates to compute

$$
d_{2}(h)=\sqrt{u_{2}-\bar{u}_{2}(h)} .
$$

Using this, we define the following two conditions which will assist us in deciding whether the aircraft gained or lost altitude.

$$
\begin{aligned}
& \mu_{1}(h)=|| h_{2: 1}(h)-h\left|-d_{2}(h)\right| \\
& \mu_{2}(h)=|| h_{2: 2}(h)-h\left|-d_{2}(h)\right|
\end{aligned}
$$

The vertical manoeuvre can be approximate if two acceptable conditions are found at a given fixed height $h\left(\sim h_{1}\right)$. The method fails when there is no solution for $\epsilon_{2}$ in the calculation of $h_{2: 1}(h)$ and $h_{2: 2}(h)$ or when the conditions are very close. This can be handled by using the previous change in 
altitude $h_{1}-h_{0}$ and in such, assuming that the vertical manoeuvre graph is smooth. We have $h_{2} \sim h+\delta_{2}(h)$ where

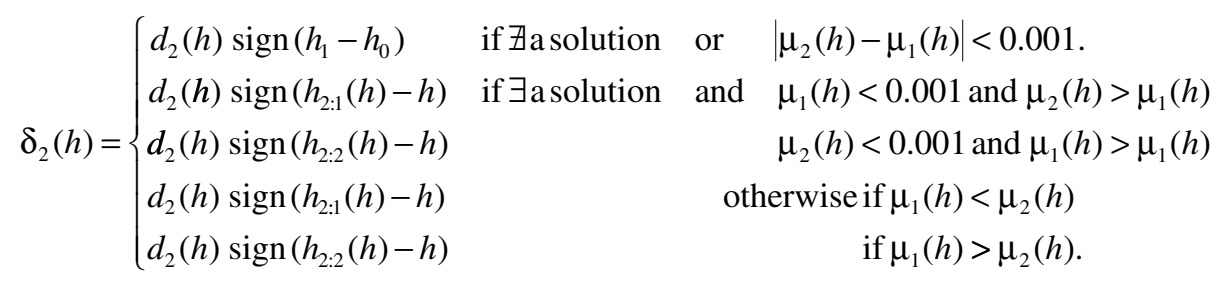

The vertical manoeuvre graphs for our three examples using this method to calculate the change in altitude are illustrated in Figure 33.7. The numbers displayed in the legends of the error graphs are of the form Calculated: Average Error (m) [Maximum Error (m)]. The errors in the beginning

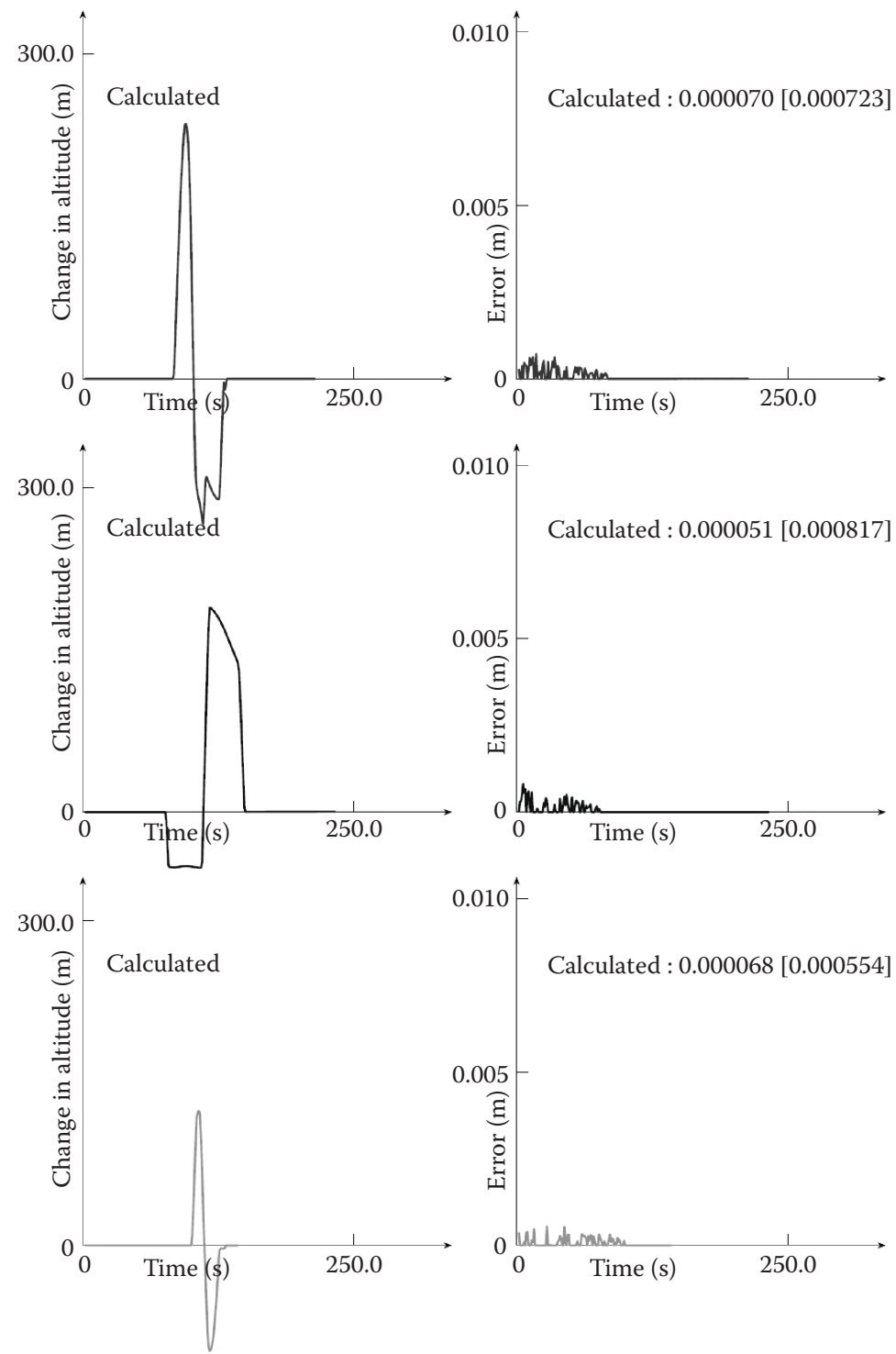

FIGURE 33.7 Vertical manoeuvre graphs. 
TABLE 33.1

Numerical Values of the Various Error-Metrics (Change in Altitude Calculation)

\begin{tabular}{lccccccc} 
& \multicolumn{3}{c}{ Sensor Offset Radius } & & \multicolumn{3}{c}{ Change in Altitude Errors } \\
\cline { 2 - 4 } Scenario & Range $[\mathrm{m}]$ & Dir $\left[{ }^{\circ}\right]$ & Altitude $[\mathrm{m}]$ & & Avg & Max & Errors \\
Pitch and dive & $0-1000$ & $0-360$ & $0-1000$ & & 0.000003 & 0.001094 & 0.000000 \\
Standard & 0 & 0 & 0 & & 0.000000 & 0.000034 & 0 \\
Max average & 330 & 260 & 110 & & 0.000015 & 0.001047 & 0 \\
Max single & 600 & 110 & 280 & & 0.000010 & 0.001094 & 0 \\
High dive & $0-1000$ & $0-360$ & $0-1000$ & & 0.000000 & 0.001126 & 0.000000 \\
Standard & 0 & 0 & 0 & & 0.000000 & 0.000001 & 0 \\
Max average & 110 & 110 & 670 & & 0.000005 & 0.001126 & 0 \\
Max single & 110 & 110 & 670 & & 0.000005 & 0.001126 & 0 \\
Long toss & $0-1000$ & $0-360$ & $0-1000$ & & 0.000003 & 50.764000 & 0.000008 \\
Standard & 0 & 0 & 0 & & 0.000000 & 0.000001 & 0 \\
Max average & 720 & 0 & 180 & & 0.357493 & 50.764000 & 1 \\
Max single & 260 & 0 & 90 & & 0.357493 & 50.764000 & 1 \\
& & & & & & &
\end{tabular}

are due to rounding errors in the calculation of $d_{2}$ giving a false indication that there was a small change in altitude when the aircraft was actually in straight flight. The whole method was set up with a threshold of $1 \mathrm{~mm}$ which suggests that we should ignore changes in altitude smaller than this threshold. By doing this, we end up with average and maximum errors of less than $0.0001 \mathrm{~mm}$. Moving the sensor away from the defended asset will affect the calculation of the vertical manoeuvre in the sense that the occasional error (not present in the standard setup) might arise. These errors refer to instances where the algorithm gave an increase in altitude when there was actually a decrease and vice versa. This is entirely dependent on the flight profile through. Table 33.1 displays the average error, maximum error and error probability (or number of errors for specific scenarios) when moving the sensor within a $1 \mathrm{~km}$ radius away from the defended asset. Table 33.1 is further expanded to include the standard and extreme scenarios where maximums are found. Figure 33.8 illustrates this for a special case. All the errors were due to the system having no solution and caused by using the previous update based on the assumption of continuous flight. Table 33.2 displays the average error, maximum error and error probability (indicating how likely the vertical manoeuvre calculation

Sensor is located $720 \mathrm{~m}$ east and $180 \mathrm{~m}$ above defended asset
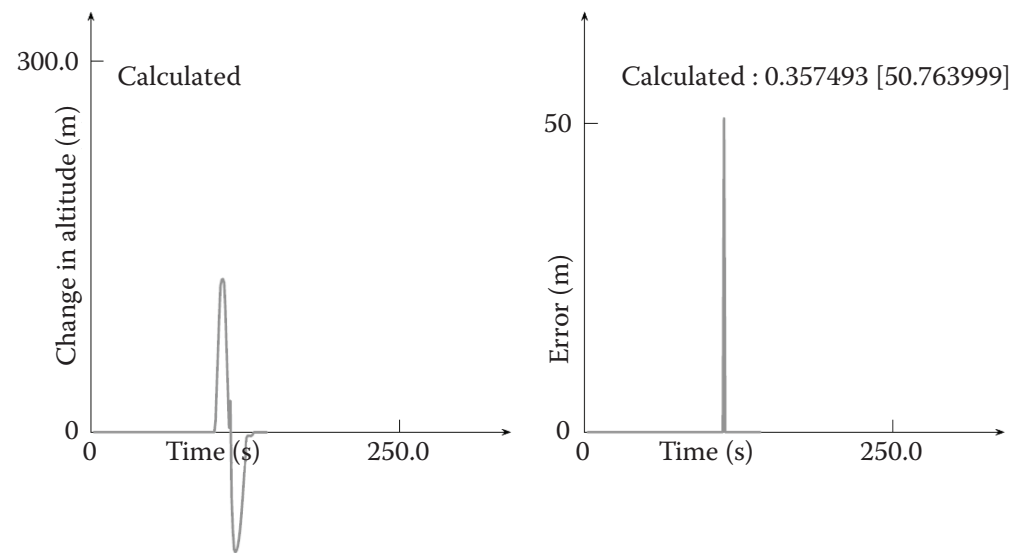

FIGURE 33.8 Vertical manoeuvre approximation. 
algorithm is to fail) for the three given profiles as the sensor moves further away from the defended asset. All vertical activity errors are still due to the system having no solution.

\subsection{CONTINUOUS TRACKING}

As aforementioned, this gives us a method to track a target in 3D from the data sets $\left(r_{1}, \theta_{1}, \hat{v}_{1}\right)$ and $\left(r_{2}, \theta_{2}, \hat{v}_{2}\right)$ accumulated from two consecutive sensor updates at time $t_{1}$ and $t_{2}$, respectively. We will refer to this method as the AACT (Altitude Approximation via Continuous Tracking) method. The method to approximate the change in altitude $h_{2}-h_{1}$ at time $t_{2}$ described in the previous section takes as input an initial value $h\left(\sim h_{1}\right)$ which is used as initial approximation. The algorithm will adjust this height before trying to compute the change in altitude to yield better conditions $\mu_{1}(h)$ and $\mu_{2}(h)$. This is done by using the given range $r_{1}$ at time $t_{1}$ to find a more accurate approximation $h \sim h_{1}$ by mapping onto the range sphere.

The AACT method uses this idea to continuously track a target in 3D by (at the $n$th sensor update) provide: (i) an estimate $\delta_{n}$ for the change in altitude $h_{n}-h_{n-1}$ by using the approximated height $h_{n-1}$ (computed at the previous sensor update) as input and (ii) an estimate for the height $h_{n}$. We will take $h_{0}$ as the AASC height whilst one may just as well take it as some other constant value (Figure 33.5). Moving the sensor away from the defended asset does affect the average AACT height errors for a specific flight profile but this is entirely dependent on the actual profile. Table 33.3 displays the average error, maximum error when moving the sensor within a $1 \mathrm{~km}$ radius away from the defended asset. The standard and extreme scenarios where maximums are found are also given. Table 33.4 displays the average error and maximum error for the AACT method for the three given profiles as the sensor moves further away from the defended asset. In the case of the above three scenarios we are better off when the sensor is $5 \mathrm{~km}$ away from the defended asset but this might not always be the case.

\subsubsection{Turning Manoeuvres}

More complex turning manoeuvres in the flight path will naturally increase the error probability when detecting vertical manoeuvres and a bad first AASC approximation might cause a scenario to

TABLE 33.2

Numerical Values of the Various Error-Metrics (for Given Profiles)

\begin{tabular}{lcccc}
\multirow{2}{*}{ Scenario } & \multicolumn{3}{c}{ Change in Altitude Errors } \\
\cline { 3 - 5 } Pitch and dive & Radius & Avg & Max & Err Prob \\
& $0-1000$ & 0.00000289 & 0.00109412 & 0.00000000 \\
& $1000-2000$ & 0.00000288 & 0.00112229 & 0.00000000 \\
High dive & $2000-3000$ & 0.00000291 & 0.00126226 & 0.00000000 \\
& $3000-4000$ & 0.00000307 & 6.53600000 & 0.00000278 \\
& $4000-5000$ & 0.00000365 & 9.44600000 & 0.00000278 \\
& $0-1000$ & 0.00000005 & 0.00112551 & 0.00000000 \\
Long toss & $1000-2000$ & 0.00000005 & 0.00113658 & 0.00000000 \\
& $2000-3000$ & 0.00000056 & 20.73200000 & 0.00000556 \\
& $3000-4000$ & 0.00000017 & 0.00154998 & 0.00000000 \\
& $4000-5000$ & 0.00000039 & 0.00150087 & 0.00000000 \\
$0-1000$ & 0.00000302 & 50.76400000 & 0.00000833 \\
& $1000-2000$ & 0.00000019 & 0.00151653 & 0.00000000 \\
& $2000-3000$ & 0.00000049 & 0.00151914 & 0.00000000 \\
& $3000-4000$ & 0.00000403 & 50.76400000 & 0.00001111 \\
& $4000-5000$ & 0.00000439 & 50.76400000 & 0.00000833
\end{tabular}


TABLE 33.3

Numerical Values of the Various Error-Metrics (Altitude Approximation/for Three Scenarios)

\begin{tabular}{lcccccc} 
& \multicolumn{3}{c}{ Sensor Offset Radius } & & \multicolumn{2}{c}{ AACT Errors } \\
\cline { 2 - 4 } Scenario & Range $[\mathrm{m}]$ & Dir $\left[{ }^{\circ}\right]$ & Altitude $[\mathrm{m}]$ & & Avg & Max \\
Pitch and dive & $0-1000$ & $0-360$ & $0-1000$ & & 0.025447 & 1.014586 \\
Standard & 0 & 0 & 0 & & 0.000020 & 0.000036 \\
Max average & 990 & 350 & 0 & & 0.252607 & 1.014428 \\
Max single & 980 & 10 & 0 & & 0.252499 & 1.014586 \\
High dive & $0-1000$ & $0-360$ & $0-1000$ & & 0.002799 & 0.014047 \\
Standard & 0 & 0 & 0 & & 0.000001 & 0.000001 \\
Max average & 980 & 60 & 0 & & 0.004149 & 0.013457 \\
Max single & 960 & 10 & 0 & & 0.004039 & 0.014047 \\
Long toss & $0-1000$ & $0-360$ & $0-1000$ & & 0.038625 & 0.546741 \\
Standard & 0 & 0 & 0 & & 0.000019 & 0.000020 \\
Max average & 990 & 350 & 0 & & 0.242730 & 0.546741 \\
Max single & 990 & 10 & & 0 & 0.242729 & 0.546741 \\
\hline
\end{tabular}

TABLE 33.4

Numerical Values of the Various Error-Metrics or the AACT Method/Altitude Approximation

\begin{tabular}{lccc} 
& & \multicolumn{2}{c}{ AACT Errors } \\
\cline { 3 - 4 } Scenario & Radius & Avg & Max \\
Pitch and dive & $0-1000$ & 0.02544727 & 1.01458580 \\
& $1000-2000$ & 0.00496901 & 0.03094935 \\
& $2000-3000$ & 0.00304537 & 0.01616566 \\
& $3000-4000$ & 0.00226141 & 0.01117682 \\
High dive & $4000-5000$ & 0.00183988 & 0.00870827 \\
& $0-1000$ & 0.00279944 & 0.01404736 \\
& $1000-2000$ & 0.00210260 & 0.00998633 \\
Long toss & $2000-3000$ & 0.00170983 & 0.00788764 \\
& $3000-4000$ & 0.00146634 & 0.00655928 \\
& $4000-5000$ & 0.00129752 & 0.00570002 \\
& $0-1000$ & 0.03862496 & 0.54674133 \\
& $1000-2000$ & 0.00890965 & 0.03202178 \\
& $2000-3000$ & 0.00527871 & 0.01690597 \\
& $3000-4000$ & 0.00377008 & 0.01177279 \\
& $4000-5000$ & 0.00294581 & 0.00909983 \\
\hline
\end{tabular}

have an average AACT height error that is above the average for the standard flight profile. The various results are shown in Figures 33.9 and 33.10, and Table 33.5.

\subsubsection{Practical Aspects}

It should be noted that the accuracy of the method to calculate a vertical manoeuvre is dependent on the accuracy to which we can measure $\left(r_{1}, \theta_{1}\right)$ and $\left(r_{2}, \theta_{2}\right)$ and $w_{2}$. It is well known that $2 \mathrm{D}$ radars 


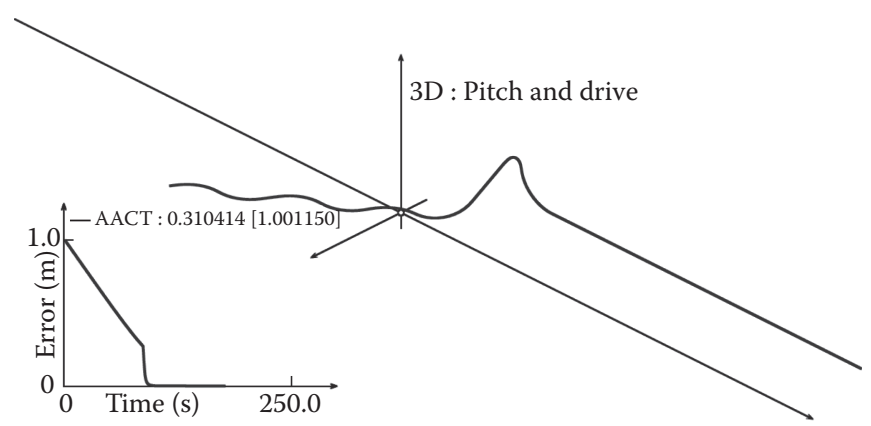

FIGURE 33.9 Height approximation using the AACT method: some results.

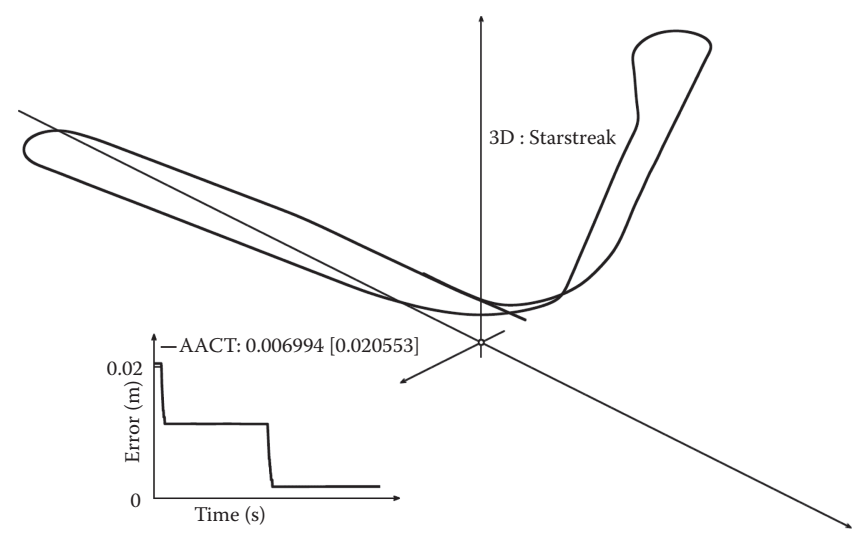

FIGURE 33.10 Height approximation using the AACT method: some more results.

TABLE 33.5

\section{Additional Flight Profiles}

\begin{tabular}{lcccccc} 
& \multicolumn{3}{c}{ Change in Altitude Errors } & & \multicolumn{2}{c}{ AACT Errors } \\
\cline { 2 - 4 } Scenario & Avg & \multicolumn{1}{c}{ Max } & Err Prob & & Avg & Max \\
Pitch and dive & 0.00248889 & 37.47600000 & 0.00248889 & & 0.03543821 & 1.03319801 \\
Stars treak & 0.00000130 & 0.00082250 & 0.00000000 & & 0.01112874 & 0.03089402 \\
\hline
\end{tabular}

have excellent slant range measurements but poor azimuth readings. The data displayed in Table 33.6 shows the effect of randomly introduced errors in azimuth readings. The errors we have encountered here w.r.t. calculation of vertical manoeuvres are not only due to the system having no solution but due to the algorithm failing as a result of the errors in azimuth readings. As one would naturally expect, average errors and error probability goes up as the azimuth error bias does.

\subsection{ALTERNATIVE METHOD}

The solution suggested in Ref. [3] can be used when no Doppler data is available. It makes use of two separate motion models that independently estimate the behaviour of the tracked entity. The relative errors between predictions of these models and observations made by the $2 \mathrm{D}$ radar are then 
TABLE 33.6

\section{Adding Random Azimuth Errors}

\begin{tabular}{|c|c|c|c|c|c|c|}
\hline \multirow[b]{2}{*}{ Scenario } & \multirow{2}{*}{$\begin{array}{c}\text { Azimuth } \\
\text { Error [mrad] }\end{array}$} & \multicolumn{3}{|c|}{ Change in Altitude Errors } & \multicolumn{2}{|c|}{ AACT Errors } \\
\hline & & Avg & Max & Err Prob & Avg & Max \\
\hline Pitch and dive & 0.0000 & 0.000003 & 0.001094 & 0.000000 & 0.025447 & 1.014586 \\
\hline \multirow[t]{5}{*}{ Radius : 0-1000 } & 0.1000 & 0.016568 & 471.120000 & 0.002519 & 0.047542 & 1.741158 \\
\hline & 0.2000 & 0.019945 & 471.120000 & 0.008628 & 0.046481 & 1.737893 \\
\hline & 0.3000 & 0.022500 & 471.120000 & 0.016542 & 0.045727 & 1.767407 \\
\hline & 0.4000 & 0.024488 & 471.120000 & 0.025508 & 0.045189 & 1.764944 \\
\hline & 0.5000 & 0.026517 & 471.120000 & 0.034939 & 0.044771 & 1.761901 \\
\hline High dive & 0.0000 & 0.000000 & 0.001126 & 0.000000 & 0.002799 & 0.014047 \\
\hline \multirow[t]{5}{*}{ Radius : 0-1000 } & 0.1000 & 0.000106 & 346.184000 & 0.000194 & 0.002799 & 0.014047 \\
\hline & 0.2000 & 0.000376 & 346.184000 & 0.000411 & 0.002819 & 0.278816 \\
\hline & 0.3000 & 0.001648 & 346.184000 & 0.000683 & 0.003003 & 0.499761 \\
\hline & 0.4000 & 0.003521 & 346.184000 & 0.000753 & 0.003489 & 0.499910 \\
\hline & 0.5000 & 0.005684 & 346.184000 & 0.000961 & 0.004194 & 0.499916 \\
\hline Long toss & 0.0000 & 0.000003 & 50.764000 & 0.000008 & 0.038625 & 0.546741 \\
\hline \multirow[t]{5}{*}{ Radius : 0-1000 } & 0.1000 & 0.083851 & 194.536000 & 0.308378 & 0.040033 & 0.546741 \\
\hline & 0.2000 & 0.132797 & 194.536000 & 0.647572 & 0.039996 & 0.546741 \\
\hline & 0.3000 & 0.175531 & 194.536000 & 0.976175 & 0.039965 & 0.546741 \\
\hline & 0.4000 & 0.214212 & 194.536000 & 1.255950 & 0.039937 & 0.546741 \\
\hline & 0.5000 & 0.249601 & 194.536000 & 1.475436 & 0.039913 & 0.546741 \\
\hline
\end{tabular}

used to make probabilistic statements on the current vertical behaviour of the aircraft. These models are based on inferring changes in the perceived velocity of a tracked target from a 2D radar track. The perceived changes may be due to changes in the target acceleration, changes in target altitude, or a combination of both. For this method to be useful we need to assume that the aircraft is at a fixed initial height $h$. We will proceed in vector notation to describe the 3D position vectors $p_{\mathrm{n}}$ (at time $t_{\mathrm{n}}$ ) obtained by projecting the actual aircraft positions onto a 2D horizontal plane at height $h$.

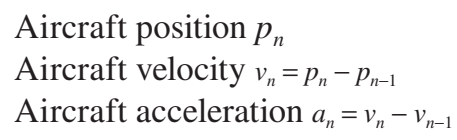

The first model assumes that the aircraft is flying at a constant altitude. This implies that perceived deviations (on the projection plane) from the expected flight path must be due to changes in velocity of the target. We can associate a $2 \mathrm{D}$ position from a single $2 \mathrm{D}$ radar sensor update via the above mentioned projection. A second update can then be used to estimate the perceived velocity of the target and finally a third update can be used to estimate the change in velocity of the target. We will not concern ourselves with the time term as it does not influence the result, provided updates occur at a constant rate. These values can then be used to predict position of the target at the next update.

$$
p_{n+1}=p_{n}+v_{n}+a_{n}
$$

The second model assumes that the estimated aircraft speed stays constant during updates thus all perceived deviations from the expected flight path are due to changes in altitude. This model additionally also assumes that the aircraft was in flying level during the prior update. Two predictions are made: one prediction for the case that the aircraft gained altitude and another for the case that the 
aircraft lost altitude. We iteratively find a new position $p_{n}$ in each of the above cases. We start with a constant step size $\delta_{0}$ which will be halved at every iteration and an initial value $q_{0}=p_{n}$ for the aircraft position. We will continue to adjust the position $q_{i}$ by increasing/decreasing its altitude by the current step size and mapping it onto the range sphere of the sensor until $\left|q_{i}-p_{n-1}\right|=\left|p_{n-1}-p_{n-2}\right|$ or the step size $\delta_{i}$ is small enough. At termination we update our position $p_{n}=q_{i}$ and make a prediction about where the target will be at the next update.

$$
p_{n+1}=p_{n}+v_{n}
$$

We can include other predictions here as the aircraft might steepen its ascent or start to level out. The predictions of these models are compared with actual observations during the subsequent sensor update. This is done by projecting the prediction to two dimensions representing slant range and azimuth. The Euclidean distance between the projected 2D position and the observed position is taken to be our measure of error. It is assumed that the smaller the error, the more likely the manoeuvre. We will abide by the following set of rules to decide as to which model to follow:

1. If the sum of all three prediction errors (representing upwards, downwards and acceleration-based errors) is less than a threshold value, then the aircraft is considered to be in straight flight. This threshold should be in the range of $5-10 \%$ of typically observed errors.

2. If the sum of all three prediction errors exceeds an upper threshold, then the aircraft is assumed to be flying a complex manoeuvre which cannot be captured by the underlying models. This threshold should be around twice the typical observed errors during normal manoeuvres.

3. If the acceleration errors is at least $10 \%$ less than the average of the upwards and downwards errors, then the aircraft is considered to be accelerating.

4. After ruling out the above three situations, we will assume that the aircraft is flying a vertical manoeuvre. The probability of the manoeuvre being upwards $\mu_{U}$ or downwards $\mu_{D}$ is given by the ratio of the corresponding errors $e_{U}$ and $e_{D}$, respectively:

$$
\begin{gathered}
\mu_{U}=\frac{e_{D}}{e_{D}+e_{U}} \\
\mu_{D}=\frac{e_{U}}{e_{D}+e_{U}}
\end{gathered}
$$

It is crucial to note that the distinction between upwards and downwards error is typically quite small thus not a lot of weight should be given to probabilistic statements with respect to vertical manoeuvres being up or down. Unfortunately, this is exactly what is needed to allow accurate continuous tracking of a target.

\subsection{CONCLUSIONS}

We have given a method to estimate vertical manoeuvres of an aircraft using a single 2D Radar when Doppler data is available and used to track a target in 3D. The average error when tracking a target in 3D using this method depends on the actual flight profile, position of the sensor w.r.t the defended asset and the accuracy of the data we get from the sensor but on average never exceeded $0.05 \mathrm{~ms}^{-1}$ for the standard flight profile examples we considered even with an $0.03^{\circ}$ error bias in the azimuth readings. The maximum error at any given time was never more than $1.8 \mathrm{~m}$. An alternative method that can be used when we do not have Doppler data was briefly outlined but this method 
statistically proven to be right only $50 \%$ of the time making it equivalent to throwing a dice when we have to decide whether the aircraft gained or lost altitude.

\section{REFERENCES}

1. D. E. Manolakis, Aircraft vertical profile prediction based on surveillance data only. In IEEE Proceedings on Radar, Sonar and Navigation, 144(5), 301-307, 1997.

2. H. Hakl, E. Davies and W.H. Le Roux, Aircraft height estimation using 2-D radar. Defence Science Journal, 60(1), 100-105, 2010.

3. H. Hakl and W.H. Le Roux, Vertical activity estimation using 2-D radar. Scientia Militaria: South African Journal of Military Studies, 36(2), 60-76, 2008. 